\title{
Multiple VHL-related hemangioblastomas and holocord syrinx: identifying the causative lesion. Illustrative case
}

\author{
Armin Mortazavi, BS, ${ }^{1}$ Diana Nwokoye, MS, ${ }^{1,2}$ David T. Asuzu, MD, PhD, ${ }^{1,2,3}$ Gretchen Scott, RN, BSN, ${ }^{1}$ \\ Panagiotis Mastorakos, MD, PhD, ${ }^{1,3}$ and Prashant Chittiboina, MD ${ }^{1,2}$

\begin{abstract}
${ }^{1}$ Surgical Neurology Branch and ${ }^{2}$ Neurosurgery Unit for Pituitary and Inheritable Diseases, National Institute of Neurological Disorders and Stroke, Bethesda, Maryland; and ${ }^{3}$ Department of Neurosurgery, University of Virginia, Charlottesville, Virginia
\end{abstract}

BACKGROUND Brainstem and spinal cord hemangioblastomas are a common manifestation of von Hippel-Lindau (VHL) disease. Cysts and associated syringes are the most common cause of significant morbidity in these patients. Surgical treatment of symptomatic hemangioblastomas are often complicated by the presence of multiple potential lesions, leading to cyst and syrinx formation.

OBSERVATIONS The authors present a case of a patient with multiple VHL-related hemangioblastomas who presented with syringobulbia and holocord syrinx. Resection of two cyst wall hemangioblastomas and one cervical hemangioblastoma only transiently improved syringobulbia. Eventual resolution of syringobulbia and collapse of the holocord syrinx only occurred following removal of a large lower thoracic hemangioblastoma.

LESSONS Surgical management of hemangioblastomas and associated cysts in patients with VHL should only target lesions most likely contributing to neurological deficits as excess surgical intervention risks treatment-related morbidity. The authors illustrate how anatomical and pathophysiological considerations as well as patient symptoms are key to identifying target lesions for resection and developing deliberate treatment plans.

https://thejns.org/doi/abs/10.3171/CASE21296

KEYWORDS VHL; von Hippel-Lindau; hemangioblastoma; syrinx; cyst

von Hippel-Lindau (VHL) disease is an autosomal dominant gene syndrome that occurs due to germline mutations to the VHL gene on chromosome $3 p .^{1}$ More than $80 \%$ of patients with VHL develop central nervous system (CNS) hemangioblastomas and of these, greater than $90 \%$ will develop multiple lesions. ${ }^{2,4}$ Hemangioblastomas associated with $\mathrm{VHL}$ are diagnosed most commonly in the second or third decade, while sporadic cases arise often in the fifth or sixth decade of life. ${ }^{1,3,5}$ In the setting of $\mathrm{VHL}, 50 \%$ of lesions occur in the spine, $40 \%$ in the cerebellum, and $10 \%$ in the brainstem. ${ }^{6}$ Natural history studies have shown that hemangioblastomas grow in a saltatory unpredictable pattern, ${ }^{7}$ and many tumors do not become symptomatic. $^{2}$ Fundamentally, resection is reserved for hemangioblastomas that become symptomatic due to tumor growth or cyst-related mass effect.
Peritumoral cysts and syringes are the most frequent cause of symptoms, and $90 \%$ of patients with symptomatic hemangioblastomas have associated syrinxes. ${ }^{3,4,8}$ Less than $50 \%$ of hemangioblastomas with associated cysts will progress to become symptomatic. ${ }^{9}$ The pathogenesis of peritumoral cysts has been studied extensively. Hemangioblastomas are characterized by increased vascular permeability and increased intratumoral pressure, which results in plasma ultrafiltrate leaking into the surrounding CNS tissue, leading to edema and ultimately cyst formation. ${ }^{9-11}$ Given that cyst formation is a direct result of the hemangioblastoma, resection of the tumor often results in resolution of the cyst without need for resection of fenestration of the cyst. ${ }^{2,9,10}$ Notably, cyst formation is not related directly to tumor size. When symptoms occur, cyst size is on average 100 times larger than the associated tumor in the cerebellum and 10-15 times larger than the associated tumor in the brain stem or

ABBREVIATIONS CNS = central nervous system; MRI = magnetic resonance imaging; $\mathrm{POD}=$ postoperative day; $\mathrm{RUE}=$ right upper extremity; $\mathrm{VHL}=$ von Hippel-Lindau.

INCLUDE WHEN CITING Published September 13, 2021; DOI: 10.3171/CASE21296.

SUBMITTED May 12, 2021. ACCEPTED July 12, 2021.

(C) 2021 The authors, CC BY-NC-ND 4.0 (http://creativecommons.org/licenses/by-nc-nd/4.0/). 

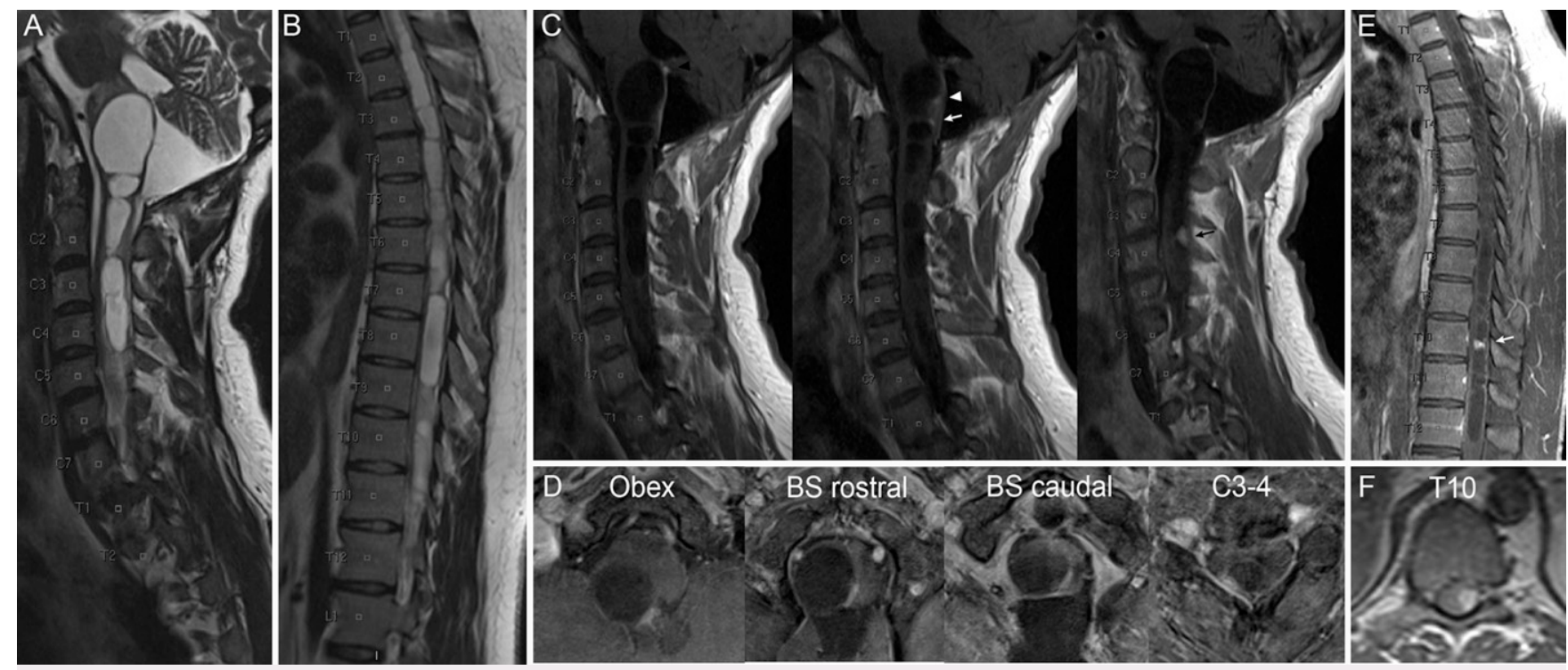

FIG. 1. Cervical and thoracic MRI at initial presentation. A and B: Cervical (A) and thoracic $(\mathbf{B})$ T2 sagittal MRI demonstrates extensive septated syringes throughout the brainstem and spinal cord. C and D: T1 postcontrast sagittal (C) and axial (D) cervical MRI demonstrates obex (black arrowhead), brainstem (BS) rostral (white arrowhead), BS caudal (white arrow), and C3-4 (black arrow) hemangioblastomas. E and F: T1 postcontrast sagittal (E) and axial (F) thoracic MRI demonstrates left intramedullary T10 hemangioblastoma.

spinal cord. ${ }^{9}$ It may be difficult to determine the primary tumor contributing to cyst formation, especially in the setting of multiple hemangioblastomas. Currently, proximity to the cyst wall is the primary method of identifying the contributing tumor and identifies the surgical target most likely to result in cyst resolution. ${ }^{8,10,11}$

Here, we present a case of a patient with multiple VHL-related hemangioblastomas who presented with syringobulbia and a holocord syrinx. The patient was primarily symptomatic from a brainstem cyst, which did not permanently resolve following resection of two brainstem cyst wall-associated hemangioblastomas and one cervical hemangioblastoma. Eventual resolution of syringobulbia and collapse of the holocord syrinx was achieved following removal of a lower thoracic hemangioblastoma. This case highlights the nuances and difficulties associated with management of syndromic patients with multiple CNS tumors as well as the techniques used for resection of intramedullary hemangioblastomas.

\section{Illustrative Case}

We report a case of a 30-year-old man with a history of $\mathrm{VHL}$ with prior resection of a large obex hemangioblastoma who presented with 2 months of worsening neck pain radiating to bilateral upper extremities with associated dense numbness of the right upper extremity (RUE). Over the prior week, he also experienced episodes of vertigo and intermittent diplopia. He noted difficulty swallowing solids as well as intermittent coughing. Neuroaxis imaging demonstrated a $30 \times 23 \times 24 \mathrm{~mm}$ cyst in the right medulla oblongata in continuity with a septated syrinx spanning from $\mathrm{C} 1$ to C4 (Fig. 1A), as well as a septated syrinx from C6 to the conus (Fig. 1B). These were associated with three small brainstem hemangioblastomas, one at the obex and the other two at the left dorsal wall of the cyst (rostral and caudal hemangioblastomas), as well as a right C3-4 hemangioblastoma adjacent to the right dorsal wall of the cervical syrinx (Fig. 1C and D). Imaging also demonstrated a $7 \times$
$7 \mathrm{~mm}$ left T10 intramedullary hemangioblastoma (Fig. 1E and F). Given these findings, the patient underwent C3-4 laminectomies for hemangioblastoma resection (Fig. 2A) and a redo suboccipital approach for resection of the rostral brainstem hemangioblastoma (Fig. 2B). Postoperative computed tomography demonstrated no complications and decreased cyst size to $28 \times 19 \times 21 \mathrm{~mm}$. Postoperatively, the patient reported improvements in pain, sensation, and swallowing. $\mathrm{He}$ was discharged on postoperative day (POD) 4. One week postdischarge, he noted recurrence of RUE numbness, difficulty swallowing, and evidence of aspiration. Magnetic resonance imaging (MRI) of the brain and cervical spine confirmed resection of the target dorsal
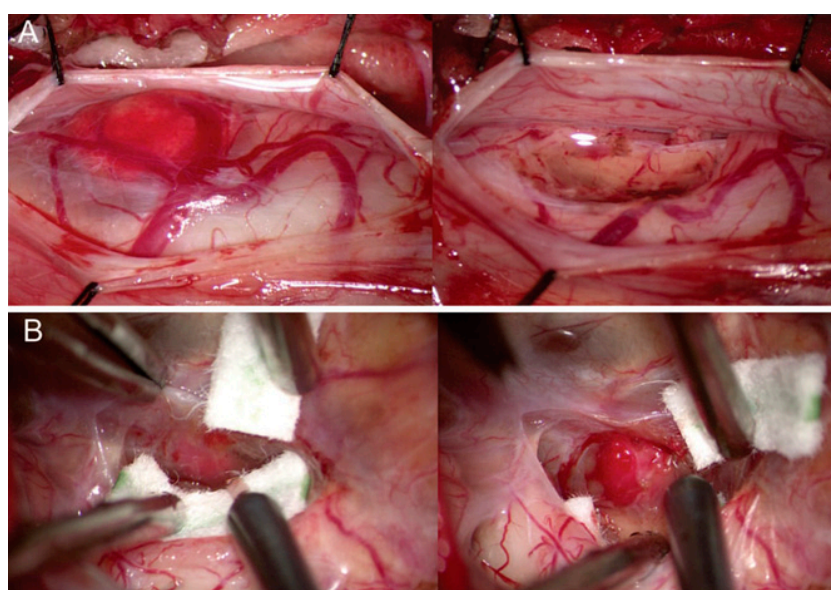

FIG. 2. First operative intervention. A: Microscopic intraoperative images demonstrating right intramedullary cervical hemangioblastoma preresection (left) and postresection (right). B: Microscopic intraoperative images demonstrating rostral brainstem hemangioblastoma on cyst wall premyelotomy (left) and postmyelotomy (right). 

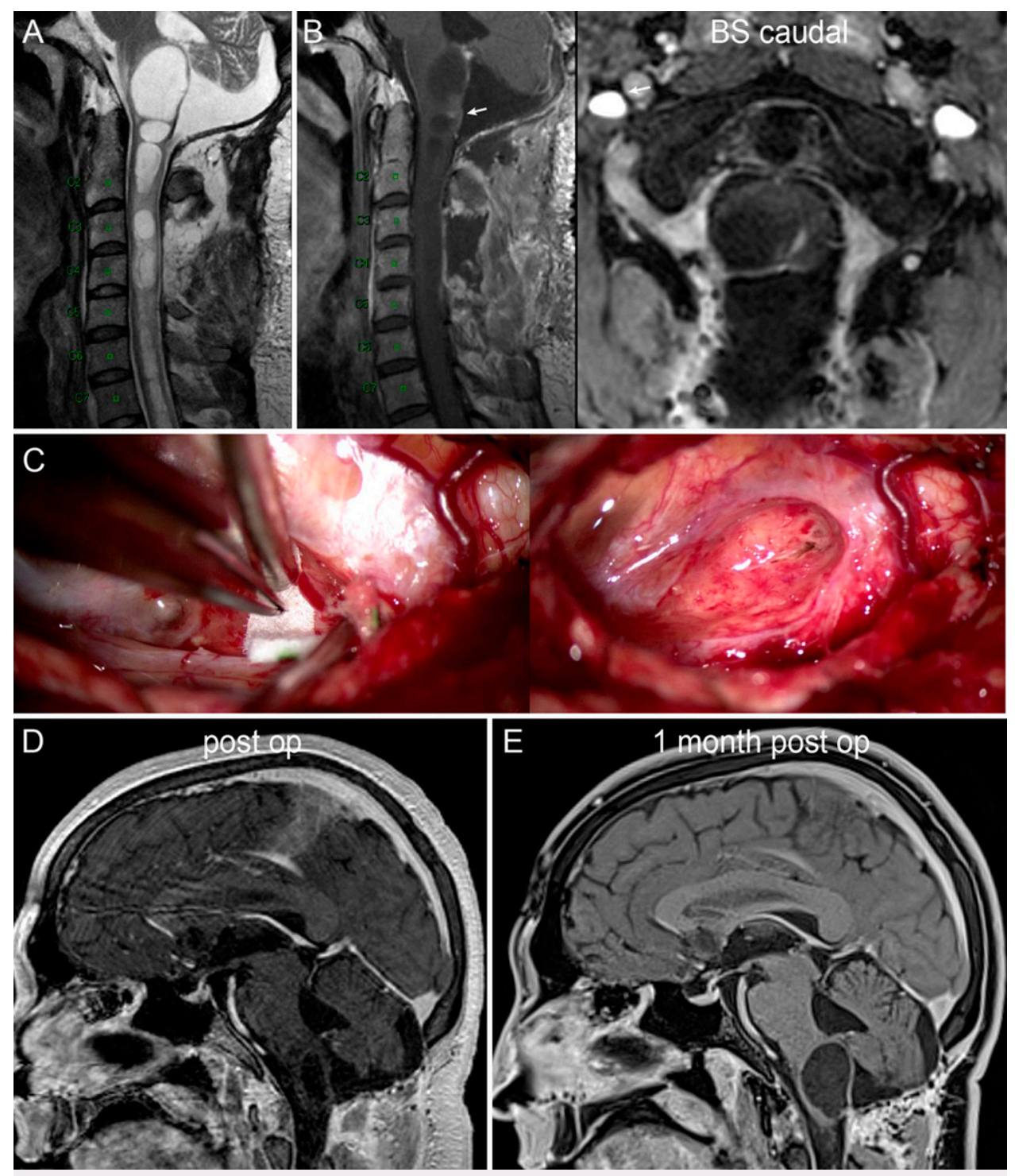

FIG. 3. Postoperative cyst recurrence and second operative intervention. A: Cervical T2 sagittal MRI demonstrates cyst recurrence. B: Cervical T1 postcontrast MRI demonstrates remaining caudal brainstem (BS) cyst wall hemangioblastoma. C: Microscopic intraoperative images demonstrating caudal BS hemangioblastoma on cyst wall preresection (left) and postresection (right). D: Postoperative T1 postcontrast brain MRI confirmed resection and drastically decreased cyst size. E: T1 postcontrast brain MRI 1-month postoperatively demonstrating cyst recurrence.

medulla hemangioblastoma and C3-4 hemangioblastoma, as well as decrease in size of the C1-4 syrinx. However, the brain stem cyst had returned to its initial preoperative size (Fig. $3 \mathrm{~A}$ and $\mathrm{B}$ ). As such, we proceeded with a redo suboccipital approach for resection of the caudal brain stem hemangioblastoma (Fig. $3 \mathrm{C}$ ). Postoperative MRI confirmed resection and dramatically decreased cyst size to $20 \times 9 \times 11$ $\mathrm{mm}$ (Fig. 3D). The patient reported symptom improvement and was discharged on POD3. One month after discharge, he experienced symptom recurrence with RUE numbness, gait instability, and difficulty swallowing. Brain MRI demonstrated recurrence of the brain stem cyst $27 \times 20 \times 18 \mathrm{~mm}$ (Fig. 3E). Given the persistence of the cyst and the presence of septated syringes with associated edema throughout the spinal cord (Fig. 4A), we hypothesized that a large $(6 \times 6 \times 6$ $\mathrm{mm}$ ) intramedullary spinal cord hemangioblastoma at T10 (Fig. 4B) was causing enough ultrafiltrate leak ${ }^{9}$ to sustain the holocord syrinx and to reinflate the synringobulbia. We proceeded with a T9-10 laminectomy for hemangioblastoma resection (Fig. 5A and Video 1).

VIDEO 1. T9-10 laminectomies for resection of T10 hemangioblastoma resection. This video demonstrates the microdissection steps for intramedullary hemangioblastoma resection, including pial cuff incision, dissection along gliotic plane, coagulation, and section of deep arterial feeders. Click here to view.

The patient noted postoperative symptom improvement, and imaging demonstrated partial collapse of the syringes throughout 

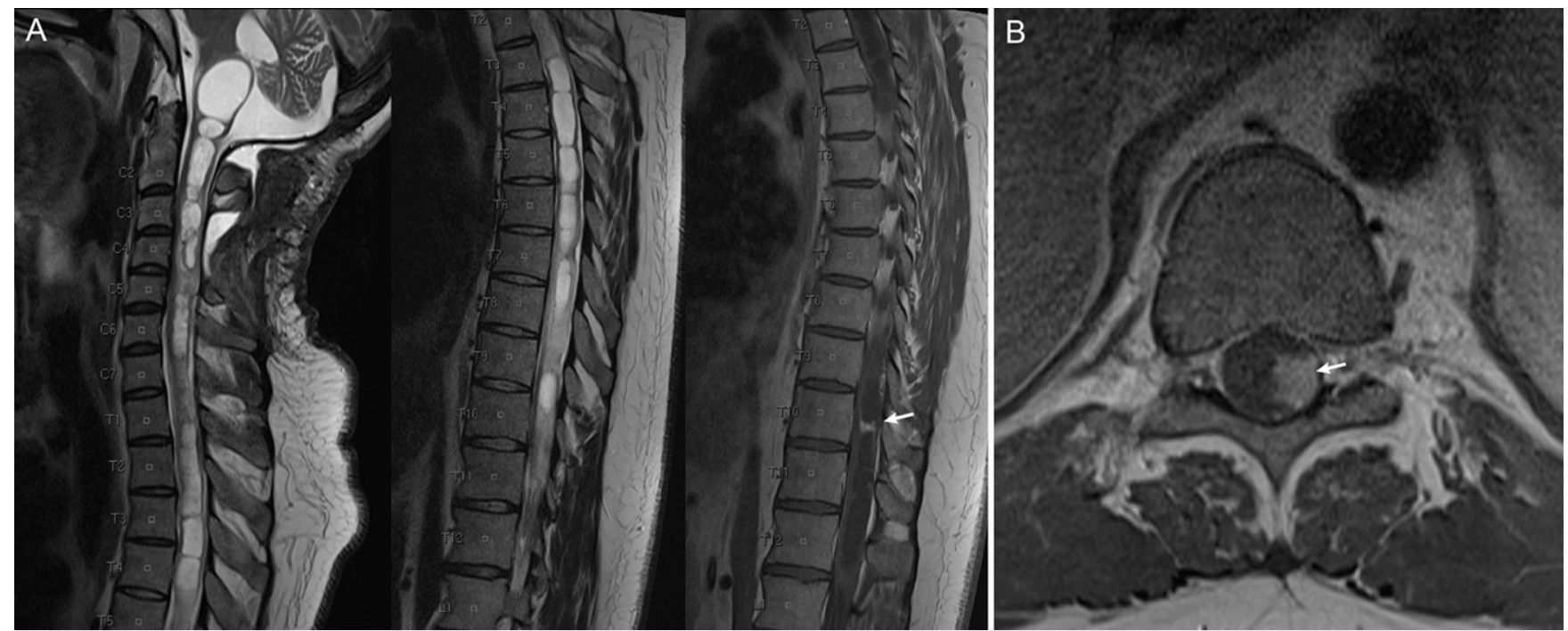

FIG. 4. Postoperative cyst recurrence. A: Cervical and thoracic T2 MRI demonstrates persistent syringes from the brainstem to the conus. T1 postcontrast thoracic MRI demonstrates a T10 hemangioblastoma (white arrow). B: T1 postcontrast axial thoracic MRI demonstrates a left intramedullary T10 hemangioblastoma (white arrow).

the spinal cord as well as a decrease in size of the brain stem cyst to $19 \times 12 \times 6 \mathrm{~mm}$ (Fig. $5 \mathrm{~B}$ ). He was discharged on POD3. At 1 year follow-up, the patient noted persistence of mild intermittent right shoulder pain as well as mild sensory deficit C6-8 but denied gait instability, difficulty swallowing, or significant sensory deficits. Neuroaxis MRI at 1 year demonstrated near-complete collapse of the syringes throughout the spinal cord (Fig. 5C). The patient has had no recurrence of symptoms, the surgically removed hemangioblastomas, or the syrinx during the follow up (13 months).
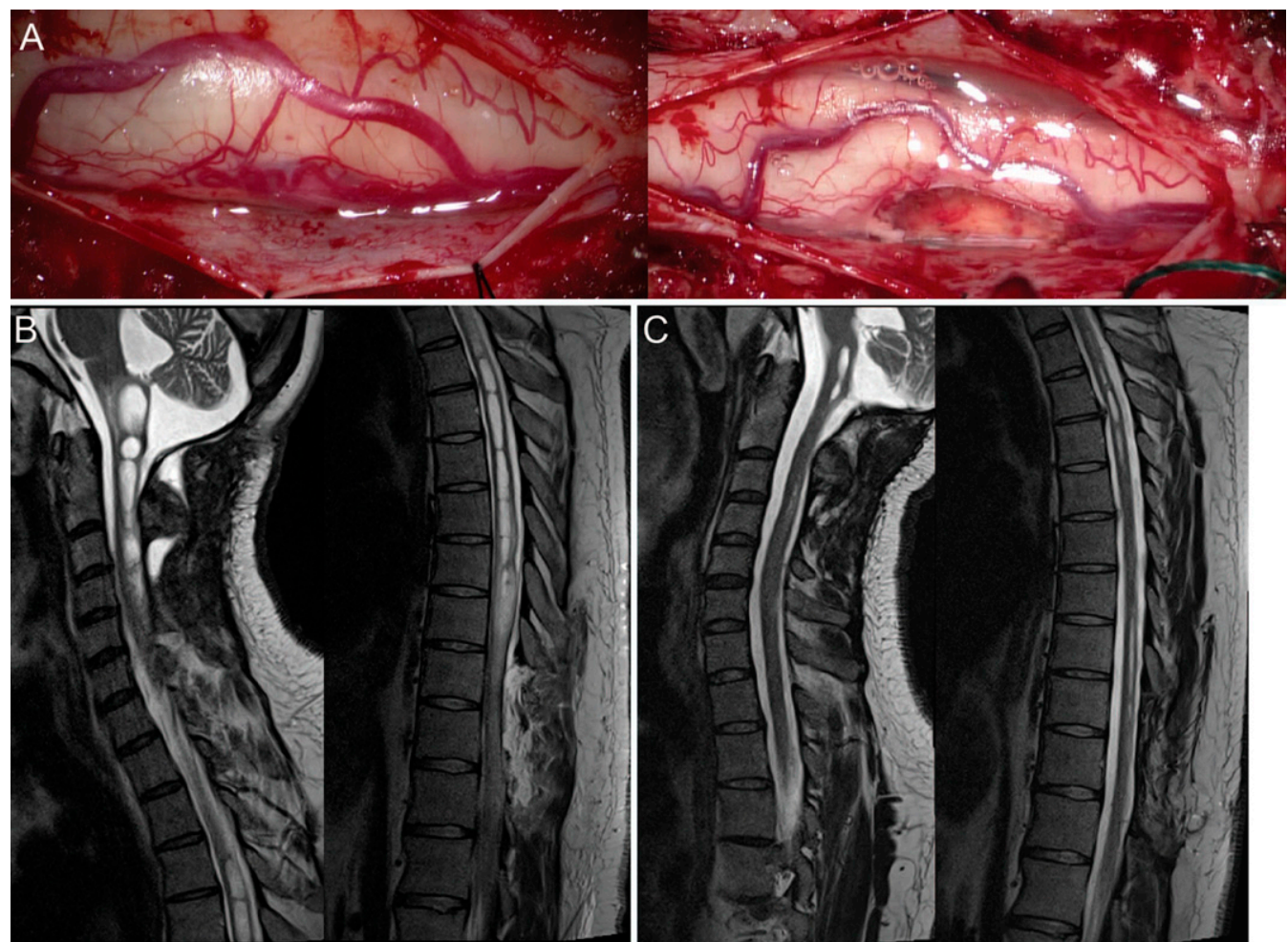

FIG. 5. Third operative intervention. A: Microscopic intraoperative images demonstrating a T10 intramedullary hemangioblastoma preresection (left) and postresection (right). B and C: T2 cervical and thoracic MRI on POD1 (B) and on 1-year follow-up (C) demonstrate gradual collapse of the syringes throughout the brainstem and spinal cord. 


\section{Discussion \\ Observations}

The primary strategy for management of VHL-related hemangioblastomas includes targeting only the symptomatic lesions to avoid treatment related morbidity. ${ }^{5}$ This case highlights the complexity and nuances associated with neurosurgical management of patients with VHL with multiple CNS tumors and syringes, especially in cases of unclear etiological association with a single hemangioblastoma.

Up to $85 \%$ of patients with VHL with hemangioblastomas have a hemangioblastoma-associated syrinx, which can span an average size of 6.9 vertebral levels. ${ }^{12}$ The extensive syringes and multiple hemangioblastomas render identification of the culprit hemangioblastoma difficult.

In this case, the patient presented with symptoms consistent with a high cervical and brainstem lesion. In the setting of multiple hemangioblastomas in proximity with the brain stem cyst and cervical syrinx, there were no distinct characteristics to aid identification of the culprit hemangioblastoma. Importantly, the size of hemangioblastomas does not correlate with cyst formation. ${ }^{10}$ Once a cyst is formed, moreover, it grows 100 -fold faster than the associated tumor. ${ }^{10}$ To this end, when patients with VHL present with neurological deficits, it is critical to obtain whole-spine MRI imaging in order to best assess the lesion burden. Furthermore, serial imaging is critical in identifying the behavior of tumors and the associated cysts/syringes.

The leaky tumor vasculature and high intratumoral pressure in hemangioblastomas leads to edema and subsequently to formation of a fluid-filled cavity. Resection of the tumor results in resolution of the cyst without need of cyst fenestration. ${ }^{2,9,10}$ In this particular case, it became evident with cyst recurrence that an unanticipated hemangioblastoma was contributing to the formation of the syrinx. We initially approached the case by removing the hemangioblastomas within the cyst wall as well as the larger cervical hemangioblastoma with an associated cervical syrinx. However, persistent cyst recurrence led to evaluation of a distant thoracic hemangioblastoma with an associated thoracolumbar syrinx. Edema often precedes syrinx formation as the ultrafiltrate from hemangioblastomas traverse the parenchyma extracellular space. ${ }^{10,11}$ As such, even in the absence of a direct cavity connection, distant hemangioblastomas can fill a distant cyst, as observed here. Collapse of the syringes from the brain stem to the conus suggests this case may be considered pathophysiologically as a holocord syrinx associated with a single hemangioblastoma, as previously reported. ${ }^{13,14}$

Multiple studies have identified factors associated with poor outcomes following resection, including tumor volume, anterior location, the presence of peritumoral edema, intramedullary tumors, and myelotomies. ${ }^{5,12,15-17}$ However, long-term follow-up of VHL-disease associated spinal cord hemangioblastomas have demonstrated that patients can remain functionally stable following resection. In a prospective natural history study of VHL reviewing 121 hemangioblastomas with associated peritumoral cysts, resection of all appropriately selected tumors universally resulted in collapse or resolution of the cyst without need for removal of the cyst wall. ${ }^{7,9}$ Thus, if lesions can be appropriately identified and safely removed, resection of spinal cord hemangioblastomas in the setting of $\mathrm{VHL}$ disease can be performed safely and effectively.

\section{Lessons}

This case illustrates the management of complex patients with VHL and multiple hemangioblastomas with associated cysts/ syringes. We illustrate how anatomical considerations and patient symptoms should be utilized in identifying target lesions for resection, which may require sequential procedures. Advances in imaging technology may help to establish the hemangioblastoma culprit for syrinx formation.

\section{Acknowledgments}

This study was supported by the Intramural Research Program of the National Institute for Neurological Diseases and Stroke.

\section{References}

1. Lonser RR, Glenn GM, Walther M, et al. von Hippel-Lindau disease. Lancet. 2003;361(9374):2059-2067.

2. Dornbos D 3rd, Kim HJ, Butman JA, Lonser RR. Review of the neurological implications of von Hippel-Lindau disease. JAMA Neurol. 2018;75(5):620-627.

3. Wanebo JE, Lonser RR, Glenn GM, Oldfield EH. The natural history of hemangioblastomas of the central nervous system in patients with von Hippel-Lindau disease. J Neurosurg. 2003;98(1):82-94.

4. Butman JA, Linehan WM, Lonser RR. Neurologic manifestations of von Hippel-Lindau disease. JAMA. 2008;300(11):1334-1342.

5. Lonser RR, Weil RJ, Wanebo JE, DeVroom HL, Oldfield EH. Surgical management of spinal cord hemangioblastomas in patients with von Hippel-Lindau disease. J Neurosurg. 2003;98(1):106-116.

6. Neumann HP, Eggert HR, Weigel K, Friedburg H, Wiestler OD, Schollmeyer P. Hemangioblastomas of the central nervous system. A 10-year study with special reference to von Hippel-Lindau syndrome. J Neurosurg. 1989;70(1):24-30.

7. Lonser RR, Butman JA, Huntoon K, et al. Prospective natural history study of central nervous system hemangioblastomas in von Hippel-Lindau disease. J Neurosurg. 2014;120(5):1055-1062.

8. Ammerman JM, Lonser RR, Dambrosia J, Butman JA, Oldfield EH. Long-term natural history of hemangioblastomas in patients with von Hippel-Lindau disease: implications for treatment. J Neurosurg. 2006;105(2):248-255.

9. Huntoon K, Wu T, Elder JB, et al. Biological and clinical impact of hemangioblastoma-associated peritumoral cysts in von Hippel-Lindau disease. J Neurosurg. 2016;124(4):971-976.

10. Lonser RR, Vortmeyer AO, Butman JA, et al. Edema is a precursor to central nervous system peritumoral cyst formation. Ann Neurol. 2005;58(3):392-399.

11. Lonser RR, Butman JA, Oldfield EH. Pathogenesis of tumor-associated syringomyelia demonstrated by peritumoral contrast material leakage. Case illustration. J Neurosurg Spine. 2006;4(5):426.

12. Mehta GU, Asthagiri AR, Bakhtian KD, Auh S, Oldfield EH, Lonser RR. Functional outcome after resection of spinal cord hemangioblastomas associated with von Hippel-Lindau disease. J Neurosurg Spine. 2010;12(3):233-242.

13. Dutta $G$, Singh $D$, Singh $H$, Srivastava AK, Jagetia A, Agrawal A. Dorsal hemangioblastoma manifesting as holocord syringomyelia. Surg Neurol Int. 2018;9:73.

14. Pai SB, Krishna KN. Secondary holocord syringomyelia with spinal hemangioblastoma: a report of two cases. Neurol India. 2003;51(1):67-68.

15. Kanno H, Yamamoto I, Nishikawa R, et al. Spinal cord hemangioblastomas in von Hippel-Lindau disease. Spinal Cord. 2009;47(6):447-452.

16. Pluta RM, luliano B, DeVroom HL, Nguyen T, Oldfield EH. Comparison of anterior and posterior surgical approaches in the treatment of ventral spinal hemangioblastomas in patients with von Hippel-Lindau disease. J Neurosurg. 2003;98(1):117-124.

17. Van Velthoven V, Reinacher PC, Klisch J, Neumann HP, Gläsker S. Treatment of intramedullary hemangioblastomas, with special 
attention to von Hippel-Lindau disease. Neurosurgery. 2003;53(6):1306-1314.

\section{Disclosures}

The authors report no conflict of interest concerning the materials or methods used in this study or the findings specified in this paper.

\section{Author Contributions}

Conception and design: Chittiboina, Mortazavi, Mastorakos. Acquisition of data: Mortazavi, Nwokoye, Scott, Mastorakos. Analysis and interpretation of data: Chittiboina, Mortazavi, Nwokoye, Asuzu, Mastorakos. Drafting the article: Chittiboina, Mortazavi, Nwokoye, Mastorakos. Critically revising the article:
Chittiboina, Mortazavi, Asuzu. Reviewed submitted version of manuscript: Chittiboina, Mortazavi, Asuzu, Scott, Mastorakos. Approved the final version of the manuscript on behalf of all authors: Chittiboina. Study supervision: Chittiboina.

\section{Supplemental Information}

Video

Video 1. https://vimeo.com/575401174

\section{Correspondence}

Prashant Chittiboina: National Institute of Neurological Disorders and Stroke, Bethesda, MD. prashant.chittiboina@nih.gov. 